\title{
Saúde Mental em Contextos Rurais: o Trabalho Psicossocial em Análise
}

\author{
Maurício Cirilo Neto \\ Magda Dimenstein \\ Universidade Federal do Rio Grande do Norte, RN, Brasil. Universidade Federal do Rio Grande do Norte, RN, Brasil.
}

\begin{abstract}
Resumo: Moradores de assentamentos de reforma agrária têm uma vida marcada por condições de vida e trabalho precárias, que, aliadas às barreiras no acesso às políticas e programas de saúde e assistência social, agravam as situações de vulnerabilidade que impactam a saúde mental. Este trabalho pretende discutir o cuidado desenvolvido por equipes de saúde e assistência social referente às demandas de saúde mental de assentados do Rio Grande do Norte, Brasil. Foram entrevistados 53 profissionais de forma individual ou por grupo de diferentes categorias profissionais. Para a análise dos resultados utilizou-se a Análise de Conteúdo como proposta para categorização dos dados. Resultados indicam que os trabalhadores vivenciam condições precárias de trabalho e que as equipes têm pouco conhecimento do território e das necessidades de saúde mental. O cuidado implementado ainda corresponde à lógica biomédica, com pouca participação dos moradores e desconsideração dos saberes e práticas de cuidado tradicionais. A atenção psicossocial não funciona de forma articulada apresentando problemas quanto ao seguimento e à continuidade de cuidados. Em função do desconhecimento das especificidades da população assentada e da fragmentação da rede de atenção psicossocial, essas equipes não conseguem acolher e responder às necessidades em saúde mental de modo a interferir nas iniquidades em saúde.
\end{abstract}

Palavras-chave: Assistência Integral à Saúde, Atenção Psicossocial, Saúde da População Rural, Serviços de Saúde Mental, Serviços de Saúde Rural.

\section{Mental Health in Rural Settings: Analyzing the Psychosocial Work}

Abstract: Dwellers of agrarian reform settlements have a life conditioned by poor living and work conditions; they have difficulties accessing health programs, social assistance and other public policies and this exacerbate their vulnerability, which has an impact on their mental health. This research investigates the care given by the health and social assistance staff, regarding the mental health demands of rural settlements of Rio Grande do Norte, Brazil. 53 experts from different professional categories were interviewed individually or in groups. For the analysis of the results we used the Content Analysis as a proposal for categorization of data. TR results indicate that the workers experience poor working conditions and that staff has little knowledge of the local conditions and of the mental health needs. The implemented health care still corresponds to the biomedical logic, with little participation of the dwellers, disregarding the traditional knowledge and practices of local health care. The psychosocial care is not well coordinated, presenting problems with follow-up and continuity. Due to the lack of specific knowledge of the specifics of the life conditions of the dwellers and the fragmentation of the psychosocial health care network, the staff does not abide and is not ready to face the mental health needs in order to change these health iniquities.

Keywords: Comprehensive Health Care, Psychosocial Attention, Mental Health Services, Rural Health, Rural Health Services. 


\title{
Salud Mental en Contextos Rurales: el Trabajo Psicosocial en Análisis
}

\begin{abstract}
Resumen: Residentes de asentamientos de reforma agraria tienen una vida marcada por condiciones de vida y trabajo precario, que unida a las barreras para tener acceso a las políticas y los programas sanitarios y de asistencia social agravan las situaciones de vulnerabilidad que tienen un impacto en la salud mental. Este artículo analiza el cuidado desarrollado por equipos de salud y atención social, relativos a las necesidades de salud mental de 9 (nueve) asentamientos rurales del Rio Grande do Norte, Brasil. Fueron entrevistados 53 (cincuenta y tres) de distintas categorías profesionales individualmente o en grupos. Utilizamos el Análisis de Contenido como propuesta para la categorización de los datos. Los resultados muestran que los trabajadores viven condiciones laborales precarias y los equipos tienen poco conocimiento del territorio y de las necesidades de salud mental. El cuidado implementado todavía corresponde a la lógica biomédica, con poca participación de los residentes y la desconsideración de los saberes y prácticas tradicionales de cuidado. En función del desconocimiento de las especificidades de la población asentada y la fragmentación de la red de atención psicosocial, esos equipos no logran acoger y responder a las necesidades de salud mental para cambiar las inequidades en salud.
\end{abstract}

Palabras clave: Atención Integral de Salud, Atención Psicosocial, Salud Rural, Servicios de Salud Mental; Servicios de Salud Rural.

\section{Introdução}

O termo "assentamento" apareceu pela primeira vez no vocabulário jurídico e sociológico no contexto da reforma agrária venezuelana em 1960 e se difundiu posteriormente para outros países. Segundo Bergamasco e Norder (1996, p. 7), os assentamentos rurais são "unidades de produção agrícola, criados por meio de políticas governamentais, visando o reordenamento do uso da terra, em benefício de trabalhadores rurais sem terra ou com pouca terra". A emergência de assentamentos rurais de reforma agrária é um acontecimento importante na história do meio rural brasileiro, pois os beneficiários do programa propiciaram o contato com a terra e a reconstrução de seus modos de vida.

Entretanto, moradores de assentamentos rurais de reforma agrária ainda vivem dificuldades relacionadas às adversidades socioambientais, ineficiência do suporte técnico-institucional à produção agrícola, à pobreza, cenário que se agrava quando se observa os assentamentos do nordeste brasileiro (Delgado, 2012; Instituto Nacional de Colonização e Reforma Agrária - Incra, 2010). Esse contexto de vulnerabilidade psicossocial e ambiental dificulta a vida e a permanência das populações no campo e está diretamente associado ao aparecimento de transtornos mentais e uso problemático do álcool (Confederação Nacional dos Trabalhadores da Agricultura - Contag, 2013; Costa,
Dimenstein, \& Leite, 2014; Pinheiro, Silva, Carneiro, Faria, \& Silva, 2009).

Dentre os impactos relacionados às condições de vulnerabilidade psicossocial e ambiental, destaca-se a incidência dos Transtornos Mentias Comuns (TMC). Focar os TMC permite compreender como o sofrimento psíquico diz respeito às condições de vida e trabalho e analisar os processos de determinação social da saúde mental. O termo Transtorno Mental Comum (Golberg, \& Huxley, 1992) refere-se aos sintomas não psicóticos como insônia, fadiga, irritabilidade, esquecimento, dificuldade de concentração e queixas somáticas, apresentando uma prevalência média estimada de $25 \%$ na população mundial (World Health Organization - WHO, 2001) e entre $20 \%$ e $56 \%$ na população brasileira (Santos, \& Siqueira, 2010).

No âmbito dessa discussão, estudos em populações rurais são escassos em nível internacional (España, 2011). No Brasil, um dos poucos estudos nesse sentido, o relatório da Confederação Nacional dos Trabalhadores na Agricultura (Contag, 2013), detectou como principais agravos os problemas de coluna, hipertensão, dores de cabeça constantes, disfunções gastrointestinais, alergia/problema de pele e insônia, ou seja, queixas que apontam para a possibilidade de sofrimento de base psicossocial que está associado às condições de vida e trabalho no campo. Quanto aos moradores de assentamentos 
rurais, a escassez de estudos e dados oficiais é ainda maior, refletindo a indiferença com que historicamente as iniciativas governamentais tratam as populações do campo.

Enquanto grupo populacional em situação de vulnerabilidade, as necessidades de saúde dos assentados vêm sendo negligenciadas pelas políticas públicas, além de enfrentarem dificuldades de acesso aos serviços de saúde e assistência social (Bergamaschi, Teles, Souza, \& Nakatami, 2012). Considerando este cenário problemático, o presente trabalho, fruto de uma dissertação de mestrado, discute o trabalho em saúde mental de equipes de saúde e assistência social voltado a moradores de assentamentos rurais. Além disso, articula a produção do cuidado psicossocial às condições de vulnerabilidade que a população enfrenta cotidianamente, com os modos de sociabilidade e convivência que marcam as relações nesses cenários, bem como com os saberes e práticas tradicionais e recursos informais que fazem parte do arsenal terapêutico dessas populações. Inicialmente as questões metodológicas referentes ao estudo são abordadas, para, em seguida, adentrar na análise sobre o cuidado em saúde mental em contextos rurais.

\section{Delineamento metodológico}

O Rio Grande do Norte (RN) possui 292 assentamentos rurais de reforma agrária. A seleção dos assentamentos seguiu os seguintes critérios de inclusão: a) assentamentos federais cadastrados no Incra; b) assentamentos com mais de cinco anos de criação pelo Incra; e c) assentamentos com o maior número de famílias cadastradas no Incra. Diante disso, nove assentamentos rurais do estado foram escolhidos. Os participantes da pesquisa foram os profissionais que compõem as equipes de saúde e assistência social de referência para essas populações.

O critério de escolha das equipes foi intencional. Diante da dificuldade de acesso aos profissionais, da necessidade de deslocamento até essas regiões, de contato com as secretarias e de agendamento das entrevistas, foi construída uma amostragem intencional, o que implicou priorizar os trabalhadores que conheciam o território, que alegaram desenvolver intervenções no campo da saúde mental e que aceitaram participar da pesquisa. Participaram 53 profissionais de diferentes categorias profissionais, sendo 13 assistentes sociais, 13 psicó- logos, 7 enfermeiros, quatro agentes comunitários de saúde, três educadores físicos, três médicos, dois fisioterapeutas, dois fonoaudiólogos, dois médicos do Programa Mais Médicos, dois nutricionistas, um advogado, um odontólogo, um pedagogo e um terapeuta ocupacional. Por compreender a intersetorialidade como componente fundamental do trabalho em uma perspectiva psicossocial, a diversidade de categorias profissionais foi contemplada.

Vale destacar os cuidados éticos, como autorização das secretarias responsáveis para a realização da investigação, apresentação aos participantes dos objetivos da pesquisa e procedimento a ser utilizado, leitura do Termo de Consentimento Livre e esclarecido (TCLE), riscos e benefícios envolvidos, além da autorização para a gravação do áudio das entrevistas.

O local das entrevistas foi definido pelos participantes após orientação sobre as condições favoráveis do ambiente para a realização da mesma. Algumas entrevistas foram realizadas na capital do estado, mais precisamente nas casas dos participantes ou em locais públicos da cidade. Outras ocorreram em espaços institucionais como secretarias e serviços de saúde e assistência social.

O primeiro instrumento utilizado foi um roteiro semiestruturado versando sobre o conhecimento das necessidades de saúde mental e do território, as estratégias de cuidado implementadas, a articulação em rede e continuidade do cuidado e os desafios para as práticas em contextos rurais. Foi aplicado de forma individual ou em grupo, obedecendo o critério dos participantes. Contudo, durante as viagens de campo, o encontro com o inusitado nesses contextos exigiu lançar mão de outra ferramenta metodológica. A observação participante foi uma segunda ferramenta complementar durante o processo de pesquisa, através da qual foi possível registrar acontecimentos durante os deslocamentos, no encontro com as comunidades rurais, na rotina de trabalho dos profissionais, momentos frutíferos na reconstrução da pesquisa, acessando experiências que não puderam emergir durante os momentos das entrevistas (Tedesco, Sade, \& Caliman, 2014).

A análise de conteúdo temático categorial de Bardin (2011) serviu de orientação para a categorização dos resultados, a partir de duas categorias previamente delimitadas: necessidades em saúde mental identificadas e cuidados em saúde mental ofertados. 


\section{Resultados}

\section{Perfil dos profissionais}

Os participantes estão inseridos em equipes da Estratégia de Saúde da Família (ESF) (25\%), Núcleo de Apoio à Saúde da Família (NASF) (23\%), Centro de Atenção Psicossocial ${ }^{1}$ (CAPS) (25\%), Centro de Referência de Assistência Social (CRAS) (23\%) e Centro de Referência Especializado de Assistência Social (CREAS) (6\%). Os trabalhadores em sua maioria são mulheres $(71 \%)$, jovens (55\%), possuem entre 23 e 30 anos, recém-formadas (55\%) e que concluíram a graduação há no máximo cinco anos. Esses resultados corroboram a literatura que aponta o campo da assistência social e da atenção primária como espaços ocupados por recém-formados, que se inserem temporariamente no campo das políticas públicas, enquanto não encontram empregos que julguem mais atrativos em termos salariais ou que estejam mais próximos as capitais e/ou grandes centros urbanos. Outros elementos funcionam como determinantes da maior prevalência de trabalhadores jovens, como o processo paulatino de reorientação da formação no ensino superior por meio de programas de extensão e estágio que tentam vincular os estudantes de graduação à saúde pública, iniciativas de atração e fixação dos profissionais recém-formados para trabalhar no Sistema Único de Saúde (SUS) e no Sistema Único de Assistência Social (SUAS), e ainda o processo de expansão e interiorização do ensino superior (Costa et al., 2013; Dantas, 2014; Gonçalves et al., 2014; Macedo, \& Dimenstein, 2012; Silveira et al., 2014).

O vínculo empregatício predominante é o contrato público temporário (85\%), efeito das mudanças de cunho neoliberal que atingiram o mundo do trabalho, marcado pela flexibilização, precarização e terceirização. Essas transformações assumem contornos diferenciados nas diversas regiões brasileiras, nos municípios de pequeno e médio porte, onde resiste mais fortemente nossa herança oligárquica, patrimonialista e assistencialista, cujos cenários de trabalho são agravados pelas gestões locais pautadas no autoritarismo e arbitrariedades.

\section{Necessidades de saúde mental detectadas nos assentamentos rurais}

A primazia das necessidades de saúde como princípio orientador das práticas de saúde é de fundamental importância (Souza, Araújo, Andrade, Franca,
\& Souza, 2014). O modo como as necessidades sociais de saúde são identificadas, compreendidas e organizadas no cotidiano dos serviços é essencial para compreender como o cuidado em saúde mental vem sendo ofertado aos moradores de assentamentos rurais. Apesar do pouco conhecimento dos problemas vivenciados pelos assentados, os participantes da investigação apontaram dificuldades que conformam um cenário bastante complexo e multifacetado.

São as equipes da ESF e CRAS que conhecem melhor os problemas de saúde mental vivenciados pela população, o que é compreensível por se tratarem da porta preferencial de entrada no SUS e no SUAS, pela maior capilaridade territorial e cobertura populacional. Os sofrimentos destacados se referem, principalmente, aos transtornos mentais considerados graves, persistentes e incapacitantes, o que evidencia que vários outros modos de sofrimento que não conformam a clientela tradicional dos serviços especializados se encontram invisibilizados. Os chamados TMC são formas de expressão do sofrimento que não são facilmente categorizadas como "doenças", apesar de constituírem uma variedade de sofrimento que pode estar associado ou não a outros agravos em saúde. A diversidade e complexidade de sua fenomenologia indicam que não há definição clara do que é "patológico", não cabendo muitas categorias diagnósticas (Brasil, 2013). Desse modo, a atenção a esses agravos depende da habilidade de fazer mediações entre a generalidade da ciência biomédica e a experiência singular, objetiva ou subjetiva, de uma pessoa que vivencia tal sofrimento (Mendes, 2015). Assim, uma clínica excessivamente orientada para a doença não será capaz de lidar com os sofrimentos que são contextuais, associados aos territórios onde as populações vivem ao longo da vida (Brasil, 2013; Mendes, 2015).

A experiência de sofrimento em áreas rurais assume determinadas singularidades relacionadas aos contextos de vida (Lima, \& Lopes, 2012). Comumente, o sofrimento está relacionado à baixa escolaridade, à maior dependência da família e às dificuldades laborais e financeiras (España, 2011). A saúde dos trabalhadores rurais é condicionada pelo processo de exploração de trabalho no campo. Está aliada a fatores sociais, raciais, de gênero, econômicos, tecnológicos, organizacionais, ao perfil de produção e consumo, além de fatores de risco de natureza física, química, biológica, mecânica e ergonômica presen-

${ }^{1}$ Encontramos dois tipos de CAPS nos municípios, CAPSI e CAPSII. 
tes nos processos de trabalho rural. Isso constitui um enorme desafio em termos de enfrentamento pelas políticas públicas. Nesse sentido, os participantes destacaram as dificuldades laborais e financeiras vivenciadas pelos assentados, elencando problemas na produção agrícola, inserção no mercado de trabalho pela via da informalidade, insuficiência da renda familiar, conformando o cenário histórico de pobreza rural em nosso país (Helfand, Rocha, \& Vinhais, 2009; Kageyama, \& Hoffmann, 2006), conforme aponta a enfermeira a seguir:

É porque não tem, não tem de jeito nenhum. Porque assim, o que dava muito trabalho a esse pessoal, por aqui, era a usina quando funcionava, mas aí a usina parou de funcionar, muitos e muitos ficaram sem trabalhar, só mesmo vivendo da bolsa escola, bolsa família, esses negócios, essas ajudas do governo e agora que está surgindo vários trabalhos porque estão implantando aquela energia eólica, né? (...). Porque o pessoal daqui, principalmente da zona rural, todos dependiam dessa usina que inclusive agora está fechada, né? Aí, pronto, muitos ficam em casa principalmente os pais de família, é a situação que eu vejo, tem uma puérpera está em casa e o marido deitado sem fazer nada (Enfermeira da ESF).

É importante pontuar que, apesar do reconhecimento das dificuldades vivenciadas nas rotinas de trabalho, há profissionais que reduzem a multidimensionalidade do processo saúde-doença por não compreender esse cenário como condicionante da saúde dos trabalhadores. Quando indagados de que forma os contextos de vida e o trabalho da população assentada estão relacionados aos problemas de saúde mental existentes, parte dos participantes não soube responder, alegando que a experiência do sofrimento independe dos contextos urbanos ou rurais, como conta a assistente social a seguir:

É normal. Normal. Que tem casos, assim que, que eu acho até mais, mais, pelo fato que eu já trabalhei em Natal, já trabalhei em outros municípios, eu acho até normal, como nos outros municípios, eu acho até que lá é menos, entendeu (Assistente social do NASF).

Os profissionais também destacam os conflitos familiares e a violência de gênero, em particular, a violência doméstica, como elementos preocupantes. Vinculam os conflitos familiares e violências às situações de vulnerabilidade e ao uso abusivo de álcool e outras drogas. Contudo, ao narrarem casos de violência de gênero, há posturas naturalizantes que contribuem para a sua reprodução, ou ainda culpabilizam a mulher por não conseguir alterar as situações vividas, desconsiderando que a problemática da violência contra as mulheres em contextos rurais é composta por uma rede complexa de elementos de ordem socioeconômica e cultural (Costa, Lopes \& Soares, 2015).

As relações entre saúde, iniquidades de gênero e ruralidades devem ser melhor compreendidas para estruturar diretrizes no campo da saúde de modo que possam se configurar como instrumentos de enfrentamento às situações de violência nesses contextos. A Política Nacional de Saúde Integral das Populações do Campo, das Florestas e das Águas (PNSIPCFA) reconhece as condicionalidades de gênero na saúde, todavia, não há diretrizes que possam ser operacionalizadas no cotidiano dos serviços, se restringindo ao fortalecimento de iniciativas que já vêm sendo implementadas, mas que não estão conseguindo interferir de modo significativo nos contextos de vida das mulheres do campo.

A utilização indiscriminada de psicotrópicos é outro tema de preocupação para alguns trabalhadores. Porém, constituiu-se um discurso contraditório, pois, apesar de reconhecer o uso exagerado de psicotrópicos como problema de saúde pública, foi indicado como uma das tecnologias de cuidado mais amplamente utilizada pelos trabalhadores no trato do sofrimento psíquico, indicando postura medicalizante por meio da prescrição abusiva e inadequada de psicotrópicos para situações cotidianas da vida e relacionadas às mazelas sociais (Zanello, \& Silva, 2012).

Em termos do uso de álcool, os profissionais consideram preocupante o número de mulheres que estão fazendo uso da substância, enquanto este é naturalizado na população masculina. Esse atravessamento de gênero diz respeito às relações de poder que condicionam os espaços ocupados por homens e mulheres. Isso produz barreiras de acesso aos serviços para mulheres que enfrentam problemas relacionados ao uso da substância. Nesse sentido, se reproduz o discurso de que o consumo de álcool é um comportamento desviante para as mulheres, principalmente quando não conseguem se adequar às normas sociais. A mulher é responsabilizada por vários problemas atrelados ao cotidiano de vida das famílias, tal como afirma a assistente social: 
A gente tem notado o aumento do consumo de álcool nas mulheres. Assim, é uma coisa mais velada, elas não assumem com tanta facilidade. (...). Mas a gente nota isso bastante com relação aos índices de negligência com crianças, violência contra idosos, de brigas em casa, de violência doméstica, que nos últimos tempos têm aparecido muito como causa disso, do alcoolismo feminino (Assistente social do CRAS).

Ainda no que concerne ao uso de substâncias psicoativas, os trabalhadores destacaram o uso de drogas ilícitas associado ao tráfico de drogas e ao aumento da violência no meio rural. A questão da violência no campo faz referência às novas configurações que o rural vem assumindo a partir das mudanças sociais, econômicas, políticas e culturais vivenciadas nos últimos anos, transformações que borram as tradicionais fronteiras entre o rural e o urbano, transferindo problemas sociais historicamente vinculados ao urbano para diferentes contextos sociais. A violência no campo vai assumindo novas facetas e representa um desafio para o seu enfrentamento pelas políticas públicas, pois, além do histórico de violência que os assentados carregam na luta pela terra, agora se deparam com novas condições que reforçam a situação de vulnerabilidade, como é destacado no seguinte relato:

Mas também, esse caso que vou te falar agora, não é apenas em P., (...), tem também um índice de drogas. Mediante essas comunidades ficarem próximas à M. e a S. do M., o que acontece, roubam em M. e vão fazer o desmanche em S. do M. Já foi detectado que jovens que moram naquela região estavam envolvidos em crimes, de roubo à mão armada e uso de drogas. Mas está havendo sim. Na semana passada eu tive a oportunidade de conversar com o senhor da comunidade, onde ele disse: "Minha filha eu estou com vontade de ir embora de lá, porque eles tão roubando as vacas da gente e a gente não pode dizer nada". Eu disse "Por que?" , ele disse "E eu vou morrer?". Então assim, há também um índice sim de drogas, assalto, furto, tráfico, absurdo (Coordenadora do CRAS).

Em relação às equipes de assistência social, as principais necessidades detectadas se referem ao cadastro, acompanhamento das condicionalidades e cancelamento do Programa Bolsa Família (PBF).
Seguem as diretrizes do SUAS que estabelecem como principal função dos serviços socioassistenciais o atendimento e acompanhamento de programas sociais, especialmente no que se refere às famílias em situação de vulnerabilidade (Couto, Yazbek, Silva, \& Raichelis, 2012; Dantas, 2014). Contudo, os participantes relataram que os moradores estão enfrentando dificuldades para acessar os serviços e programas socioassistenciais em razão da ausência de transporte coletivo e das precárias condições econômicas que impossibilitam a locomoção dos moradores até o centro do município. Logo, se entende que, na organização dos programas em questão, não se problematiza as barreiras de acesso enfrentadas pelos moradores, revelando a lógica institucional que desconsidera as singularidades locais, reforçando as vulnerabilidades sociais dos grupos populacionais rurais.

As dificuldades no acompanhamento das famílias beneficiárias, tal como alertou uma das equipes ao indicar que não havia família do assentamento cadastrada no serviço, são semelhantes ao que foi observado por Dias e Passos (2015) e Jesus (2011). Os autores destacam o desconhecimento dos beneficiários do programa e dificuldades de acompanhamento pelas equipes de CRAS, especialmente de moradores de contextos rurais em comparação com os dos centros urbanos. O acompanhamento das famílias beneficiárias e a inserção dos moradores nos programas e serviços socioassistenciais podem contribuir no combate às situações de vulnerabilidade ao atacar os multideterminantes associados a estas condições. As dificuldades enfrentadas na inserção dos moradores, para além dos programas de transferência de renda, desvelam heranças históricas da assistência social que podem servir como ferramenta na manutenção das situações de desigualdade social, submissão e tutela das populações.

É preciso destacar que as necessidades referidas foram significadas de diferentes modos pelos participantes. Nesse sentido, é possível distinguir dois grupos que pautam seu trabalho sob lógicas distintas. Para um primeiro grupo, as necessidades sociais e de saúde não têm relações com os problemas de saúde mental identificados. Como consequência dessa perspectiva, há uma naturalização das condições de vida e dos problemas de saúde, invizibilização dos fatores socioambientais que interferem nessas condições. Tal concepção desemboca em ações restritas aos agravos à saúde, limitando o trabalho ao conjunto de 
sintomas. Essa é uma característica do modelo tecnoassistencial marcado por uma assistência individualista e curativista, que incentiva a procura do serviço para restabelecimento da saúde por parte dos usuários, criando um ciclo de oferta e demanda interminável (Franco, \& Merhy, 2013).

No segundo grupo, os participantes consideram que as necessidades identificadas estão intimamente vinculadas aos sofrimentos vivenciados pelos moradores. Nota-se a complexificação das respostadas dadas, bem como a responsabilização ética e política diante das condições de vida da população. Nesse sentido, Ayres (2014) considera que é preciso superar as perspectivas que fatoram o processo saúde-doença das populações em vulnerabilidade, pois a complexidade dos processos implicados nas condições de saúde exige outros modos de compreensão que não sejam embasados no paradigma biomédico hegemônico. $\mathrm{O}$ autor aposta em arranjos tecnoassistenciais sensíveis às diversidades de necessidades de saúde e, portanto, potentes para responder adequadamente a elas.

No âmbito dessa discussão, é preciso destacar nossa lente compreensiva sobre os processos de determinação social da saúde e do cuidado em saúde, ambos fruto de um processo histórico, cultural, multideterminado e complexo (Almeida-Filho, 2010; Bosi, Melo, Carvalho, Ximenes, \& Godoy, 2014; Breilh, 2010; Fleury-Teixeira, \& Bronzo, 2010; Garbois, Sodre, Dalbello-Araújo, 2014).

\section{Modos de acolhimento e cuidados ofertados em saúde mental}

O acolhimento aos problemas de saúde mental vivenciados pela população assentada se baseia no modo prescritivo queixa-conduta, uma vez que as necessidades de saúde têm como resposta padrão o encaminhamento para consulta médica ou para o nível especializado. Na situação a seguir, apesar de reconhecer problemas no que se refere ao uso problemático de álcool, a profissional assume uma postura desresponsabilizada, relatando o itinerário de cuidado, da médica para o serviço especializado, anulando as possibilidades de cuidado no próprio território:

Bom, surgir questão de álcool aqui, eu mesma, nunca atendi. Eu sei assim, são conversas dos usuários que falam: "fulano de tal tão nova já tá no bar bebendo...", "fulana de tal, tão nova, filha num sei de quem, o pai alcoólatra, deixa os filhos e fica lá bebendo...". São situações assim, de atender só a médica, mas eu nunca atendi. (...). Não, conheço não, porque quando acontece geralmente passa pela médica e ela encaminha para o CAPS. (...). O CAPS aqui não funciona vinte e quatro horas vai começar agora (Enfermeira da ESF).

Compreender o acolhimento como dispositivo para reorganização do processo de trabalho implica em interferir nos encaminhamentos para a consulta médica ou especializada de forma indiscriminada, contribuindo para vincular as necessidades dos usuários às diversas possibilidades de cuidado no território. Ao desfocar da função do médico como único protagonista do cuidado, amplia-se a clínica envolvendo diversos profissionais, incluindo diferentes abordagens e olhares distintos sobre os processos de adoecimento. No contexto dessa pesquisa, o agente comunitário de saúde vem assumindo papel central no acolhimento. É um profissional que vem contribuindo na reelaboração das necessidades, na sua tradução para os códigos científicos e facilitação de uma relação dialógica com a equipe. Observou-se a desresponsabilização de muitos profissionais com a saúde mental, sobrecarregando o trabalho dos agentes, que se veem solitários na tentativa de acolher problemas que em diversos momentos escapam à competência técnica.

Quando questionados sobre o cuidado ofertado aos problemas de TMC e uso problemático do álcool, os profissionais elencam como principais estratégias a consulta individual, visita domiciliar, prescrição de medicamentos, orientação em saúde, palestras educativas. Em serviços como CAPS, CRAS e CREAS, há ainda o desenvolvimento de atividades como grupos terapêuticos, oficinas de arte, grupos de convivência e fortalecimento de vínculos, grupos socioeducativos, dentre outros, normalmente com baixa ou quase nenhuma participação de moradores dos assentamentos rurais.

A visita domiciliar e a busca ativa são tecnologias de cuidado que se destacam no arsenal de ferramentas dos trabalhadores. Essas iniciativas estão ocorrendo diante dos casos mais problemáticos, quando é solicitado pelo agente comunitário ou outro profissional, pela encomenda de programas como o de vacinação, ou ainda pelo recebimento de alguma denúncia de situações de violência, negligência, conflito na comunidade, dando um direcionamento puramente técnico e de resolução de problemas para a ação 
desenvolvida. Como indica o psicólogo, as visitas já estão definidas a partir de problemas previamente identificados, o que impossibilita apreender outras necessidades de saúde:

Não, assim, para fazer esse questionamento sobre essa questão do uso de álcool e outras drogas, depende muito da demanda que quando a gente vai para essa visita, já tem um breve diagnóstico do que é que a gente está indo fazer (Psicólogo do NASF).

As inúmeras potencialidades da visita domiciliar como ação que permite conhecer o território, estabelecer vínculos com a comunidade, identificar os problemas vivenciados pelas famílias, mapear as necessidades sociais e de saúde e realizar diagnóstico comunitário não estão sendo efetivadas no cotidiano dos serviços.

Em relação às equipes de assistência social, a busca ativa representa uma ferramenta fundamental para encontrar grupos populacionais em condições de extrema vulnerabilidade e que se encontram desassistidos pelos serviços e programas socioassistenciais. Com o advento do programa Brasil Sem Miséria, a busca ativa foi impulsionada pela criação de equipes volantes da assistência social, que se referem às equipes ligadas ao CRAS no atendimento de famílias que vivem em locais de difícil acesso ou que estão dispersas no território, como comunidades rurais ou tradicionais. No cenário da pesquisa, alguns participantes consideram a necessidade de desenvolver ações mais próximas dos assentamentos rurais, condizentes com as diretrizes do SUAS, que têm no respeito às características socioterritoriais, uma abordagem para a organização da atenção. Em um dos serviços as ações itinerantes, próximas dos moradores, estavam ocorrendo de modo sistematizado, o que lhe garantiu o nome de "CRAS Rural".

Contudo, é preciso salientar que as dificuldades encontradas pelos participantes na garantia de seu funcionamento, como reduzido número de profissionais na equipe e carência de recursos materiais, não a configuram como uma equipe volante nos termos da política de assistência social (Brasil, 2014). De todo modo, na avaliação dos trabalhadores, as ações desenvolvidas vêm possibilitando o conhecimento dos problemas vivenciados pelos moradores, bem como as potencialidades existentes no território, de modo que para os profissionais, as atividades estão produzindo interferência no cotidiano da comunidade:
Aí, quando a gente vai para as comunidades, a gente leva o atendimento social e psicológico, a mesma estrutura daqui. Se precisar de algum retorno a gente marca, se precisar de algum encaminhamento a gente envia. Normalmente a gente gosta de levar também o bolsa família, porque como só existe a sede na cidade, esse pessoal ao redor sempre tem de se deslocar para lá, dependendo das condições, né, que a gente trabalha em parceria com a saúde e educação, normalmente as escolas ou os postos de saúde cedem o espaço para a gente funcionar. Aí dependendo das condições, se tiver algum acesso a telefone, se pegar algum modem de internet, se a escola tiver algum serviço de internet, a gente já leva o bolsa família, para que seja possível fazer essas atualizações, algum cadastro novo. São os mesmos atendimentos como existem aqui no CRAS né? Tirando os serviços de convivência. Aí antes de começar, antes de fazer essa abertura, a gente já tenta levar um pouco sobre o que é o CRAS, que tipo de atividade a gente oferece, quais profissionais que nós temos (Assistente Social do CRAS).

Já as atividades de educação em saúde são valorizadas pelos participantes em razão de seu foco na promoção da saúde. Observa-se que as ações implementadas pelos participantes se restringem à realização de palestras e orientação direcionadas aos usuários. Para uma enfermeira da ESF, a almejada produção de saúde se encontra inibida, pois os moradores não se interessam por essa atividade. As temáticas das palestras são previamente escolhidas a partir de assuntos já consagrados no campo da saúde pública como saúde na gravidez, as atividades são realizadas com uso de metodologias tradicionais que desconsideram as necessidades dos usuários, sua participação, os saberes e iniciativas locais.

As ações de orientação e aconselhamento se destacam quando se trata do cuidado ao usuário problemático de álcool. A atuação dos profissionais está sendo pautada, sobretudo, pela perspectiva da abstinência. Não se nota preocupação com os contextos de vida associados ao consumo problemático de álcool. Assim, as iniciativas de cuidado são sustentadas por uma perspectiva normatizadora camuflada de promoção e prevenção de saúde, com claras intenções de controle das populações, tendo em vista modos instituídos e idealizados de saúde. Vale destacar que esta 
perspectiva, longe de instituir um espaço de acolhimento e cuidado, leva os usuários a não reconhecer tais equipes como espaço de acolhimento e cuidado, como relata o enfermeiro do CAPS quando explica que parte dos usuários não procura o serviço por acreditar que atuam pela perspectiva proibicionista no que se refere ao uso de álcool e outras drogas:

Aqui no município, até porque, assim, é estigmatizado quem usa e o CAPS. Porque a população acredita que a gente não faz redução de danos, aqui a gente tem que proibir a pessoa de usar (Enfermeiro do CAPS).

No âmbito da discussão sobre o uso problemático do álcool, há tímidas ações pautadas por perspectivas que apostam no fortalecimento da vida, respeitando os modos diferenciados de "caminhar na vida" de cada sujeito. Em vista disso, alguns trabalhadores vêm atuando na perspectiva da redução de danos, deslocando o olhar sobre o uso problemático para a existência concreta de vida dos sujeitos, reafirmando intervenções orientadas para a minimização dos danos à saúde, sociais e econômicos relacionados ao consumo de álcool sem necessariamente proibi-lo (Alves, 2009).

Observa-se que o processo de trabalho executado pelas equipes não consegue romper com os especialismos que caracterizam o modo de cuidado hegemônico no campo das políticas sociais. É preocupante que equipes de NASF, concebidas com o objetivo de arrebentar as lógicas disciplinares por meio de um trabalho baseado na interdisciplinaridade e na articulação do cuidado entre os serviços estejam reclusas em salas realizando atividades ambulatoriais, puramente assistenciais, sem nenhum contato com as equipes de saúde da família ou com a realidade dos territórios.

Entretanto, há iniciativas de compor estratégias conectadas com as necessidades da população. A equipe de um CRAS, por exemplo, diante da constatação da ausência de retaguarda especializada na região, indica que determinados grupos acompanhados pela equipe estão acolhendo o sofrimento de ordem psicossocial, causando certo desconforto por ser considerada atividade clínica não adequada ao CRAS. O discurso do profissional faz referência aos processos de determinação social da saúde, quando considera que as dificuldades socioeconômicas e as violações de direitos básicos não estão dissociadas das experiências de sofrimento vivenciadas pelos usuários:
Infelizmente ainda estamos naquela, bitolados de ficar cada um no seu dispositivo, uma coisa que eu acho uma grande ferramenta aqui no CRAS é o trabalho com grupos, que a gente consegue reunir um grupo maior de pessoas e aí trabalhar temas diversos, dando oportunidade fala e está trocando informações, não com fins terapêuticos, mas que acaba sendo terapêutico também. Então eu acho que uma das grandes ferramentas do CRAS hoje em relação a esse apoio em saúde mental são os grupos, eles atuando de forma positiva no bem-estar psíquico de cada um que vem aqui, que se coloca, chora, se posiciona, acho isso muito bacana (Assistente Social do CRAS).

Além das lógicas que sustentam e direcionam o cuidado, é importante levar em conta a sua condução nas redes de atenção à saúde. Como porta de entrada preferencial no SUS, as equipes da ESF estão enfrentando problemas no que concerne ao acolhimento e à produção de cuidados voltados para as demandas em saúde mental. Os trabalhadores comumente reclamam da pouca habilidade e competência para abordar usuários portadores de transtorno mental comum e que fazem uso problemático de álcool. O discurso dos trabalhadores sobre a loucura ainda é fortemente atrelado à periculosidade, o que tem levado uma equipe a não prestar acolhimento. A principal resposta dada diante desses casos é o encaminhamento para o nível especializado, procedimento que produz sobrecarga nos serviços do tipo CAPS, problemas que poderiam ser trabalhados pelas equipes de atenção primária, como defende a psicóloga daquele serviço:

Hoje teve um caso bem assim, que a gente vê como as pessoas ainda tão distante de compreender a rede, a RAPS. Essa senhora, ela tinha todas as características, os sintomas, tudo de um caso que era para ser acompanhado no CAPS, ela nunca veio ao CAPS. Ela fazia todo o acompanhamento na ESF, só com a renovação das receitas. E no discurso dela, ela colocava que há muito tempo estava tomando aquela medicação e que não estava fazendo efeito. (...). Então assim, um caso que era para ser acompanhado pelo CAPS, a ESF não envia, e vários casos que não eram para ser acompanhados pelo CAPS, a gente diz "Olha, a pessoa pode estar sendo matriciada daí, daí da estratégia", mandam para cá! Então são coisas 
que a gente vê que ainda está difícil trabalhar. Esse entendimento da RAPS, que a saúde mental ela pode e deve ter começado a trabalhar na saúde básica! (Psicóloga do CAPS).

Em relação ao apoio matricial realizado pelas equipes de NASF, estratégia adotada pelo Ministério da Saúde para fortalecer as ações de saúde mental na atenção primária, observa-se que não vem contribuindo, ao menos de maneira satisfatória, para a ampliação das habilidades e competências dos profissionais. O matriciamento vem se limitando a uma perspectiva assistencial, com atendimentos pontuais e de natureza ambulatorial; discussões dos casos mais problemáticos; visita domiciliar quando extremamente necessária. Os atendimentos e intervenções conjuntas entre os trabalhadores do NASF e ESF junto ao usuário, família e comunidade não faz parte do cotidiano dos serviços. Os problemas detectados estão relacionados à saturação das demandas; dificuldades para organizar e mudar o estilo de trabalho; bem como a problemas de gestão nos serviços tais como: gestão da agenda de trabalho, priorização da assistência e pressão por produção, além do privilégio de práticas biologicistas, tecnicistas, individualistas em detrimento de atenção comunitária (Paulon, \& Neves, 2013).

A proposta de coordenação do cuidado não vem sendo implementada pela atenção primária, pois ao encaminhar para outros serviços, não se acompanha o itinerário percorrido pelo usuário na rede. O sistema de referência e contrarreferência apresenta problemas de comunicação entre os serviços, e apesar dos profissionais ressaltarem que a comunicação é facilitada pela proximidade e redes de amizade, quando realizam encaminhamento, é comum não receber notícias sobre as intervenções realizadas.

Em relação ao CAPS, equipamento central da Rede de Atenção Psicossocial, não está operando na perspectiva territorial e comunitária, a qual sustenta a Estratégia de Atenção Psicossocial (EAPS). Nota-se o "encapsulamento" dos usuários que não encontram porta de saída dos serviços. Isso produz efeitos para os usuários das comunidades rurais, como conta a psicóloga do serviço, ao discutir que os usuários por não encontrarem nenhuma perspectiva de acolhimento e tratamento próximos de seu local de moradia, terminam morando no centro da cidade como maneira de garantir o acompanhamento psicossocial:
Tem um específico que está aqui desde a fundação do CAPS porque quando ela recebe alta entra em crise e para de tomar a medicação para poder voltar, e a gente tem a imensa dificuldade de fazer com essa criatura, porque eu entrei agora, mas a gente acaba vendo no prontuário que ela é de I., da zona rural, e quando ela recebe alta daqui ela volta para a zona rural, ela fica aqui na cidade para receber os cuidados, e aí ela acaba voltando. Alguns fazem isso, vem morar na cidade porque é mais fácil de conseguir atendimento (Psicóloga do CAPS).

Para responder à complexidade dos problemas de saúde vivenciados pelos moradores, os profissionais necessitam acionar a rede intersetorial de cuidado. Contudo, a lógica de referência sem contrarreferência e a inexistência de atividades pelas equipes se agrava quando envolve diferentes setores das políticas públicas. Conceber o processo de determinação da saúde exige a transformação dos serviços na busca pela intersetorialidade do cuidado. Desse modo, o reposicionamento dos processos de trabalho, a constituição de uma rede de cuidados intersetorial e a prática da interdisciplinaridade tornam-se, logo, premissas para compor práticas aliadas a esta nova concepção (Ferro, Oliveira, Silva, Zimmermann, \& Castanharo, 2015).

Diante das barreiras de acesso às redes de atenção, bem como da sua baixa capacidade de resposta e resolutividade aos problemas de saúde, os moradores utilizam seus próprios recursos para lidar com o sofrimento. Mesmo sendo alvo de processos de marginalização social, as populações rurais mantêm saberes e práticas que são produtores de pertencimento e que organizam os seus modos de compreender e agir na vida. $\mathrm{O}$ aspecto cultural, as crenças populares, religiosidade, alimentação e sociabilidades, estrutura modos próprios de responder aos processos de adoecimento, servindo para amenizar as dores e sofrimentos (Budó, \& Saupe, 2005).

Frente a estas práticas de cuidado, os profissionais tentam assumir uma postura de neutralidade, denunciando a dificuldade que os modos institucionalizados de cuidado têm em sintonizar e criar conexões com as práticas populares de saúde, como as práticas culturais e comunitárias que servem de referência aos povos rurais (Escola de Saúde Pública de Minas Gerais, 2014; Lara, Brito, \& Rezende; 2012). Nessa perspectiva, Costa, Dimenstein e Leite (2015) afirmam que é fundamental para os profissionais, 
detentores de saberes práticos e científicos, compreender estes códigos para que não sejam criadas novas barreiras entre serviços e usuários. Perante as dificuldades das equipes de acessar as comunidades rurais, a potencialização das práticas culturais e informais de cuidado torna-se foco de ação importante para os profissionais que atuam nesses contextos, tendo em vista que estas são as primeiras iniciativas tomadas pelos usuários frente aos processos de adoecimento (Silva, Dimenstein, \& Leite, 2013). Além disso, potencializar as práticas e saberes populares significa tornar os sujeitos protagonistas de suas próprias vidas.

Estabelecer modos de cuidado territorializados e culturais também envolve o âmbito comunitário. A comunidade representa para a atenção psicossocial o principal foco de investimento das ações em saúde. Assim, os cuidados elaborados pelos trabalhadores podem agir no sentido de subsidiar as iniciativas comunitárias e servir de suporte e apoio aos sujeitos. Juliano e Yunes (2014) concebem as redes de apoio social como coletivos de sistemas e pessoas significativas, que compõem os elos de relacionamentos recebidos e percebidos pelos sujeitos. A afetividade é peça crucial, em função do incontestável valor do vínculo de afeto para a constituição e manutenção das redes de apoio. Diante das condições de pobreza e vulnerabilidade que afetam as populações rurais, as redes de apoio podem se expressar como mecanismos de enfrentamento às condições adversas de vida, bem como de proteção social.

Em relação aos usuários que fazem uso abusivo de álcool, o encaminhamento indiscriminado para unidades de internação como as comunidades terapêuticas onera ainda mais as famílias que se encontram em situação de vulnerabilidade, ou o próprio município, pois em um deles a gestão está pagando por leitos de internação, reforçando a lógica hospitalocêntrica e privatista em detrimento do atendimento psicossocial, territorializado e público. Esse cenário problemático é similar ao encontrado por Silva et al. (2013) no sertão paraibano. Os autores constataram que, no momento em que os usuários necessitam de serviços de atendimento à crise, invariavelmente o serviço utilizado pelos sujeitos em sofrimento psíquico é o de internação psiquiátrica, o que representa um retrocesso para o andamento da Reforma Psiquiátrica e da Luta Antimanicomial.

A produção do cuidado nesses cenários comumente ocorre por meio da aplicação de programas verticalizados e descontextualizados, oriundos dos ministérios responsáveis, bem como pela transposição de uma lógica de cuidado construída e pensada para ambientes urbanos, que quando reproduzida em contextos rurais, não consegue responder aos problemas detectados, constituindo serviços com baixa resolutividade.

\section{Considerações finais: o trabalho psicossocial em análise}

As iniciativas políticas e institucionais recentes que tentam reorientar a atenção às populações rurais, como a PNSIPCFA, ainda não produziram resultados expressivos. Esse estudo identificou que, apesar de ações que tentam viabilizar uma atenção integral às populações assentadas, como por exemplo, equipes de saúde da família mais próximas do território, as ações em saúde são pontuais, fragmentadas e não apresentam continuidade. As equipes de saúde e assistência social vivenciam dificuldades no que se refere aos recursos humanos e materiais, os trabalhadores estão submetidos a regimes de trabalho precários e ainda atuam sob uma perspectiva biomédica.

Esse cenário é reflexo dos problemas de articulação entre contextos rurais e atenção psicossocial. Na PNSIPCFA não há diretrizes que alterem a lógica de atenção em saúde mental em vigor. Diante das necessidades de saúde mental das populações assentadas, as equipes recorrem à segurança de práticas etnocêntricas, medicalizantes, cristalizadas e com pouca capacidade resolutiva. É necessário que a atenção psicossocial efetive seus princípios de desconstrução dos saberes e práticas tradicionais e que a PNSIPCFA efetive-se como uma política com capacidade de reorientar programas, serviços e equipes na atenção à saúde integral dos povos do campo, e não apenas mais uma política que tenta acatar tensionamentos feitos por movimentos sociais do campo.

A produção do cuidado em saúde mental em contextos rurais revela uma diversidade de determinantes que se apresentam como desafios para a atenção à saúde das populações rurais e que devem ser revistas pelas políticas públicas de saúde e assistência social. Além disso, há subsídios teóricos-metodológicos que podem auxiliar na produção de cuidados que considerem os modos de vida e trabalho no campo em detrimento da atenção à saúde mental marcada pela tecnificação, objetificação, biologicismo, mecanicismo, hospitalocentrismo, curativismo e espe- 
cialismo. Diante da literatura insuficiente sobre o cuidado em saúde mental em contextos rurais e da precariedade de políticas de saúde mental sensíveis às ruralidades existentes, aponta-se para a necessidade de ampliar a caixa teórico-metodológica desse campo de modo a subsidiar a produção de cuidados numa perspectiva psicossocial, territorializada e contextual.

Este trabalho se limitou a investigar a atenção às populações assentadas, o que foi um desafio em ter- mos de aproximação da realidade de vida e trabalho dos moradores e dos profissionais. É urgente a necessidade de pesquisas que investiguem a saúde mental nos contextos rurais, com instrumentos teórico-metodológicos que respeitem os seus modos de vida, sociabilidades e modos de existência. Nesse sentido, o debate sobre a determinação social da saúde e da perspectiva territorial do cuidado fornece pistas que podem contribuir para investigações frutíferas nesses cenários.

\section{Referências}

Almeida-Filho, N. (2010). A problemática teórica da determinação social da saúde. In N. Nogueira (Org.), Determinação social da saúde e reforma sanitária (pp. 13-36). Rio de Janeiro, RJ: CEBES.

Alves, V. S. (2009). Modelos de atenção à saúde de usuários de álcool e outras drogas: discursos políticos, saberes e práticas. Cadernos de Saúde Pública, 25(11), 2309-2319. https://doi.org/10.1590/S0102-311X2009001100002.

Ayres, J. R. (2014). Vulnerabilidade, direitos humanos e cuidado: aportes conceituais. In S. Barros, P. F. S. Campos, \& J. J. S. Fernandes (Orgs.), Atenção à saúde de populações vulneráveis (pp. 1-25). Barueri, SP: Manole.

Bardin, L. (2011). Análise de conteúdo. São Paulo, SP: Edições 70.

Bergamaschi,F.P.R.,Teles, S.A.,Souza,A.C.S.\&Nakatami,A.Y.K. (2012). Reflexõesacerca daintegralidadenasreformas sanitáriaeagrária.Texto\&ContextoEnfermagem,21(3)667-674.https://doi.org/10.1590/S0104-07072012000300023

Bergamasco, S. M. P., \& Norder, L. A. (1996). O que são assentamentos rurais. São Paulo, SP: Brasiliense.

Bosi, M. L. M., Melo, A. K. D. S., Carvalho, L. B., Ximenes, V. M., \& Godoy, M. G. C. (2014). Determinantes sociais em saúde (mental): analisando uma experiência não governamental sob a ótica de atores implicados. Revista Brasileira de Epidemiologia, 17( supl. 2):126-135. https://do.org/10.1890/1809-4503201400060011

Brasil. Ministério da Saúde. (2013). Saúde mental (Cadernos de Atenção Básica, n. 34). Brasília, DF: Ministério da Saúde.

Brasil. Ministério do Desenvolvimento Social e Combate à Fome. (2014). O Brasil sem miséria. Brasília, DF: Ministério do Desenvolvimento Social e Combate à Fome.

Breilh, J. (2010). Las tres 'S'de la determinación de la vida: 10 tesis hacia una visión crítica de la determinación social de la vida y la salud. In N. Nogueira (Org.), Determinação social da saúde e reforma sanitária (pp. 87-125). Rio de Janeiro, RJ: CEBES.

Budó, M. D. L. D., \& Saupe, R. (2005). Modos de cuidar em comunidades rurais: a cultura permeando o cuidado de enfermagem. Texto \& Contexto Enfermagem, 14(2), 177-85. https://doi.org/10.1590/S0104-07072005000200004.

Confederação Nacional dos Trabalhadores da Agricultura - Contag. (2013). Relatório da Escuta Itinerante: acesso dos povos do Campo e da Floresta ao SUS. Núcleo Bandeirante: o autor. Recuperado de http://www.contag.org. $\mathrm{br} /$ index.php?modulo=portal\&acao=interna\&codpag=101\&id=9668\&data $=11 / 06 / 2014 \& \mathrm{nw}=1 \& \mathrm{mt}=1 \& \mathrm{in}=1$

Costa, M. C., Lopes, M. J. M., \& Soares, J. S. F. (2015). Violência contra mulheres rurais: gênero e ações de saúde. Escola Anna Nery, 19(1), 162-168. https://dx.doi.org/10.5935/1414-8145.20150022

Costa, M. G. S. G., Dimenstein, M., \& Leite, J. F. (2014). Condições de vida, gênero e saúde mental entre trabalhadoras rurais assentadas. Estudos de Psicologia (Natal), 19(2), 145-154. https://doi.org/10.1590/S1413-294X2014000200007

Costa, M. G., Dimenstein, M., \& Leite, J. F. (2015). Estratégias de cuidado e suporte em saúde mental entre mulheres assentadas. Revista Colombiana de Psicologia, 24(1): 13-28. https://doi.org/10.15446/rcp.v24n1.41968.

Costa, S. M., Prado, M. C. M., Andrade, T. N., Araújo, E. P. P., Silva Júnior, W. S., Gomes Filho, Z. C. et al. (2013). Perfil do profissional de nível superior nas equipes da Estratégia Saúde da Família em Montes Claros, Minas Gerais, Brasil. Revista Brasileira de Medicina de Família e Comunidade, 8(27), 90-96. httpd://doi.org/10.5712/rbmfc8(27)530

Couto, B. R., Yazbek, M. C., Silva, M. O. S., Raichelis, R. (2012). Sistema Único de Assistência Social no Brasil: uma realidade em movimento. (3a ed.). São Paulo, SP: Cortez. 
Dantas, C. M. B. (2014). A ação do psicólogo no cotidiano da assistência social: "interiorização da profissão" e combate à pobreza (Tese de doutorado). Natal: Universidade Federal do Rio Grande do Norte.

Delgado, G. (2012). Questão agrária e saúde. Rio de Janeiro, RJ: CEBES.

Dias, R. M. D. S. B., \& Passos, I. C. F. (2015). A implementação do Programa Bolsa Familia no nível local: a experiência do município de Divinópolis/MG. Revista Pesquisas e Práticas Psicossociais, 9(2), 156-172.

Escola de Saúde Pública de Minas Gerais. (2014). Cuidados em saúde mental: diálogos ntre o MST e o SUS. Belo Horizonte, MG: o autor.

España. Ministerio de Sanidad y Politica Social. (2011). Rehabilitación psicosocial de pesonas con trastorno mental grave: pautas de intervención en el domicilio y programas para contextos rurales. Castilla-La Mancha, España: Ministerio de Sanidad y Politica Social.

Ferro, L. F., Oliveira, F. R. L., Silva, E. C., Zimmermann, A. B., \& Castanharo, R. C. T. (2015). Interdisciplinaridade e intersetorialidade na estratégia de saúde da família e no núcleo de apoio à saúde da família: potencialidades e desafios. Tempus Actas de Saúde Coletiva, 8(4), 111-129. https://doi.org/10.18569/tempus.v8i4.1746

Fleury-Teixeira, P. \& Bronzo, C. (2010). Determinação social da saúde e política. In N. Nogueira (Org.), Determinação social da saúde e reforma sanitária (pp. 37-59). Rio de Janeiro, RJ: CEBES.

Franco, T. B. \& Merhy, E. E. (2013). A produção imaginária da demanda e o processo de trabalho em saúde. In T. B. Franco, \& E. E. Merhy, Trabalho, produção do cuidado e subjetividades em saúde (pp. 199-213). São Paulo, SP: Hucitec.

Garbois, J. A., Sodré, F., \& Dalbello-Araujo, M. (2014). Determinantes sociais da saúde: o "social" em questão. Saúde e Sociedade, 23(4), 1173-1182. https://doi.org/10.1590/S0104-12902014000400005

Golberg, D., \& Huxley, P. (1992) Common mental disorders: a bio-social model. London: Tavistock.

Gonçalves, C. R., Cruz, M. T. D., Oliveira, M. P., Morais, A. J. D., Moreira, K. S., Rodrigues, C. A. Q. et al. (2014). Recursos humanos: fator crítico para as redes de atenção à saúde. Revista Saúde em Debate, 38(100), 26-34. httpd://doi.org/10.5935/0103-104.20140012

Helfand, S. M., Rocha, R. \& Vinhais, H. E. (2009). Pobreza e desigualdade de renda no Brasil rural: uma análise da queda recente. Pesquisa e Planejamento Econômico, 39(1), 59-80.

Instituto Nacional de Colonização e Reforma Agrária - Incra. (2010). Reforma agrária: pesquisa sobre a qualidade de vida, produção e renda dos assentamentos da reforma agrária: relatório de pesquisa/2010. Brasília, DF: Ministério do Desenvolvimento Agrário.

Jesus, A. C. S. (2011). O programa bolsa família: impactos econômicos, socioculturais e políticos em pequenos e médios municípios do Rio Grande do Norte/Brasil (Tese de doutorado). São Luís: Universidade Federal do Maranhão.

Juliano, M. C. C., \&Yunes, M. A. M. (2014). Reflexões sobre rede de apoio social como mecanismo de proteção e promoção de resiliência. Ambiente \& Sociedade, 17(3), 135-154. https://doi.org/10.1590/S1414-753X2014000300009.

Kageyama, A. \& Hoffmann, R. (2006). Pobreza no Brasil: uma perspectiva multidimensional. Economia e Sociedade, 15(1), 26.

Lara, M. O., Brito, M.J. M., Rezende, L.C. (2012). Aspectos culturais das práticas dosAgentes Comunitários de Saúde emáreas rurais. Rev Revista da Escola de Enfermagem da USP, 46(3), 673-80. https://doi.org/10.1590/S0080-62342012000300020

Lima, K. K. S., \& Lopes, P. F. M. (2012). A qualidade sócioambiental em assentamentos rurais do Rio Grande do Norte, Brasil. Ciência Rural, 42(12), 2295-2300. https://doi.org/10.1590/S0103-84782012005000144

Macedo, J. P., \& Dimenstein, M. (2011). Expansão e interiorização da Psicologia: reorganização do saberes e poderes na atualidade. Psicologia: Ciência e Profissão, 31(2), 296-313. https://doi.org/10.1590/S1414-98932011000200008

Mendes, E. V. (2015). A construção social da atenção primária à saúde. Brasília, DF: Conselho Nacional de Secretários de Saúde.

Paulon, S., \& Neves, R. (2013). Saúde mental na atenção básica: a territorialização do cuidado. Porto Alegre, RS: Sulina.

Pinheiro, T. M., Silva, J. M., Carneiro, F., Faria, H., \& Silva, E. (2009) Saúde no campo. In A. Miranda (Coord.), Cadernos de textos da $1^{a}$ Conferencia Nacional de Saúde Ambiental (pp. 25-29). Brasília: Abrasco.

Santos, E. G., \&Siqueira, M.M. (2010). Prevalência dos transtornos mentais na população adulta brasileira:uma revisão sistemática de 1997 a 2009. Jornal Brasileiro dePsiquiatria, 59(3), 238-246. https://doi.org/10.1590/S0047-20852010000300011 
Silva, V. H F., Dimenstein, M., \& Leite, J. F. (2013). O cuidado em saúde mental em zonas rurais. Mental, 10(19), $267-285$.

Silveira, D. S., Corrêa, M. S., Saes, M. O., Kantorski, L., Jardim, V., \& Rosa, C. Q. (2014). Composição das equipes de centros de atenção psicossocial da região Sul do Brasil. Revista de Enfermagem da UFSM, 4(3), 509-518. https://doi.org/10.5902/2179769210013

Souza, M. C. D., Araujo, T. M. D., Andrade, F. A. D., Franca, A. D. J., \& Souza, J. N. (2014). Necessidades de saúde e produção do cuidado em uma unidade de saúde em um município do nordeste, Brasil. O Mundo da Saúde, 38(2), 139-148. https://doi.org/10.15343/0104-7809.20143802139148

Tedesco, S. H., Sade, C. \& Caliman, L. V. (2014). A entrevista na pista cartográfica: a experiência do dizer. In E. Passos, V. Kastrup, \& S. Tedesco (Orgs), Pistas do método da cartografia: a experiência da pesquisa e o plano do comum (Vol 2, pp. 92-127). Porto Alegre, RS: Sulina.

World Health Organization - WHO. (2001). The world health report-2001: mental health: new understanding, new hope. Geneva: o autor. Recuperado de http://www.who.int/whr/2001/en/whr01_en.pdf?ua=

Zanello, V., \& Silva, R. M. C. (2012). Saúde mental, gênero e violência estrutural. Revista Bioética, 20(2): $267-79$.

\section{Maurício Cirilo Neto}

Psicólogo. Mestre em Psicologia pela Universidade Federal do Rio Grande do Norte, Natal - RN, Brasil.

E-mail: mauricioneto_2@msn.com

\section{Magda Dimenstein}

Professora Titular do Departamento de Psicologia da Universidade Federal do Rio Grande do Norte, Natal - RN, Brasil. E-mail: mgdimenstein@gmail.com

Endereço para envio de correspondência:

Universidade Federal do Rio Grande do Norte. Programa de Pós-Graduação em Psicologia.

Campus Universitário. Lagoa Nova. CEP 59078-970.

Natal - RN, Brasil.

Recebido 13/07/2016

Reformulação 23/02/2017

Aprovado 02/05/2017

Received 07/13/2016

Reformulated 02/23/2017

Approved 05/02/2017

Recebido 13/07/2016

Reformulado 23/02/2017

Aceptado 02/05/2017

Como citar: Cirilo Neto, M., \& Dimenstein, M. (2017). Saúde mental em contextos rurais: o trabalho psicossocial em análise. Psicologia: Ciência e Profissão, 37(2), 461-474. https://doi.org/10.1590/1982-3703002542016

How to cite: Cirilo Neto, M., \& Dimenstein, M. (2017). Mental health in rural settings: analyzing the psychosocial work. Psicologia: Ciência e Profissão, 37(2), 461-474. https://doi.org/10.1590/1982-3703002542016 